\title{
DIAGNOSIS AND TREATMENT OF CONGENITAL DISLOCATION OF THE HIP JOINT IN THE NEW-BORN
}

\author{
SOPHUS von Rosen, Malmö, Sweden \\ From the Orthopaedic Clinic, Malmö General Hospital
}

The city of Malmö has about 220,000 inhabitants, and more than 99 per cent of all the children are born in the city's one obstetrical department. Since 1952 the routine paediatric examination, performed within two days and again seven days after birth, has included the hip joints. The well known snapping or clicking phenomenon of the hip has been regarded as a symptom of congenital dislocation (Froelich 1911, Le Damany 1912, Ortolani 1935, and others).

From 1952 up to June 1960 thirty-nine cases of congenital dislocation were discovered in this way. Among these cases was one of teratological disorder in a girl with multiple deformities and dislocation of one hip with the femoral head so high that it could not be reduced.

One case was missed in 1956. This was in a girl who had asphyxial convulsions the day after birth and was transferred to the Children's Hospital as an emergency. For this reason she escaped the routine examination and dislocation of the left hip was discovered only when she began to walk at one year. This case included, the total incidence was forty cases in about 24,000 births, or $1 \cdot 7$ per thousand.

The diagnosis was at first made through the " clicking phenomenon," which in all our cases was caused by the femoral head slipping in and out of the joint cavity. The clinical diagnosis was verified by radiography. This is reliable when performed with the femoral head dislocated and with the legs fully rotated inwards and each abducted 45 degrees; the long axis of the femoral shaft then points towards the anterior superior iliac process (Andrén and von Rosen 1958) (Fig. 1). When the head is reduced, however, the long axis points towards the lateral margin of the acetabulum (Fig. 2). It should be emphasised that the radiograph must be made with the femoral head dislocated because in congenital dislocation the head slips in or out of the acetabulum very easily according to the position of the legs or the active movements of the baby.

\section{TREATMENT}

Since 1956 our treatment has been to put the child in a special splint (Fig. 3) with the legs in the position of abduction and lateral rotation, the position in which the head of the femur is reduced. The splint is made from thin malleable strips of aluminium and has a rubber covering. The child can be tended by the mother and washed without being taken out of the splint.

The hip is examined every two or three weeks in the out-patient department of the clinic. When the doctor is satisfied that the reduction is stable the mother is allowed to take the child out of the splint for general care and bathing. As a rule this temporary freedom from splintage is permitted after four to eight weeks. Usually splintage is discontinued altogether before the end of the third month. In no case over the last four years has the splint been used continuously throughout the day for more than three months. In a few cases it has been used as a night splint for a short period. Redislocation has not occurred after this method of treatment. 


\section{RESULTS}

With the exception of the teratological case with multiple deformities, the results have been excellent. All the children have been kept under observation and up to the present only three of the dislocated joints fail to show normal radiographs (Figs. 4 to 7 ). These three

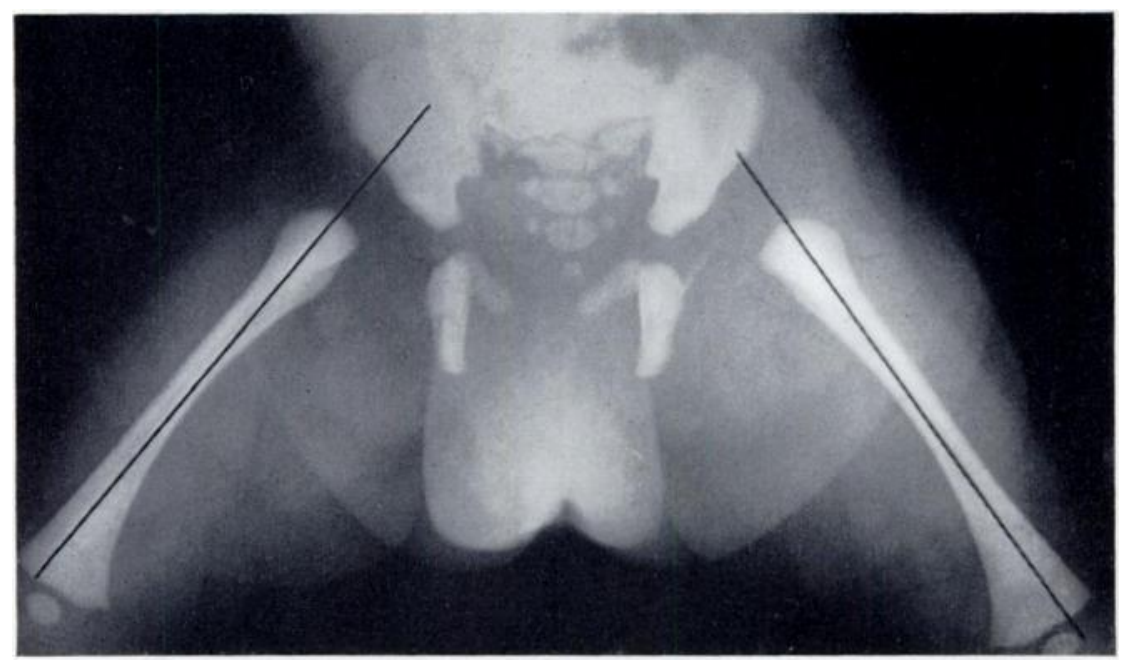

Fig. 1

Radiograph of a new-born girl with bilateral dislocation. The legs were abducted about 45 degrees and medially rotated during the exposure. The long axis of the shaft of each femur points towards the anterior superior iliac spine.

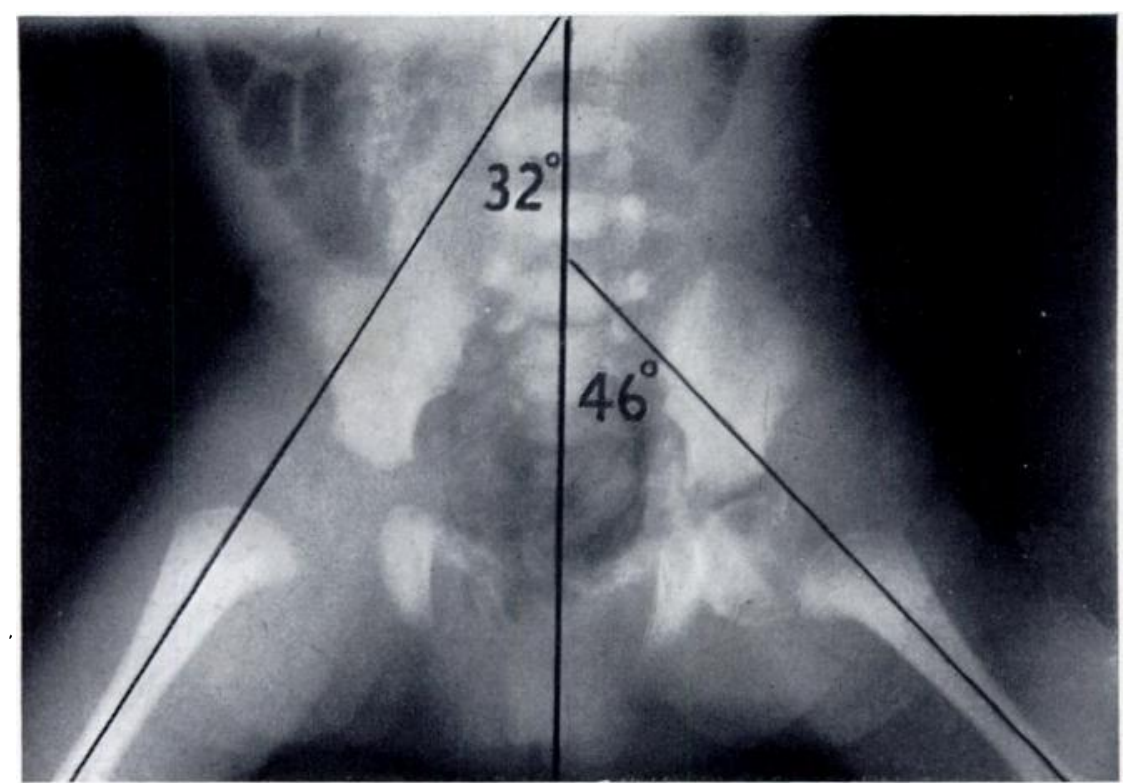

FiG. 2

Post-mortem radiograph (arthrograph on the left side) of the normal hips of a new-born child. The picture was taken in the position of medial rotation and abduction. On the left side the axis of the shaft of the femur is pointing towards the lateral margin of the acetabulum. The right side shows that in abduction less than 45 degrees the radiograph can give a false impression of dislocation.

cases are among the first eight cases, in whom the joints were not effectively and continuously held reduced. At that time we used abduction cushions or splints of various types which were removed each time the mother tended the child. Two of the children had to undergo closed

VOL. 44 B, NO. 2, MAY 1962 
reduction and plaster fixation in the standard way at five and eight months respectively. It should be pointed out, however, that steady improvement has been seen in the radiographs of these three cases (Figs. 8 to 10).

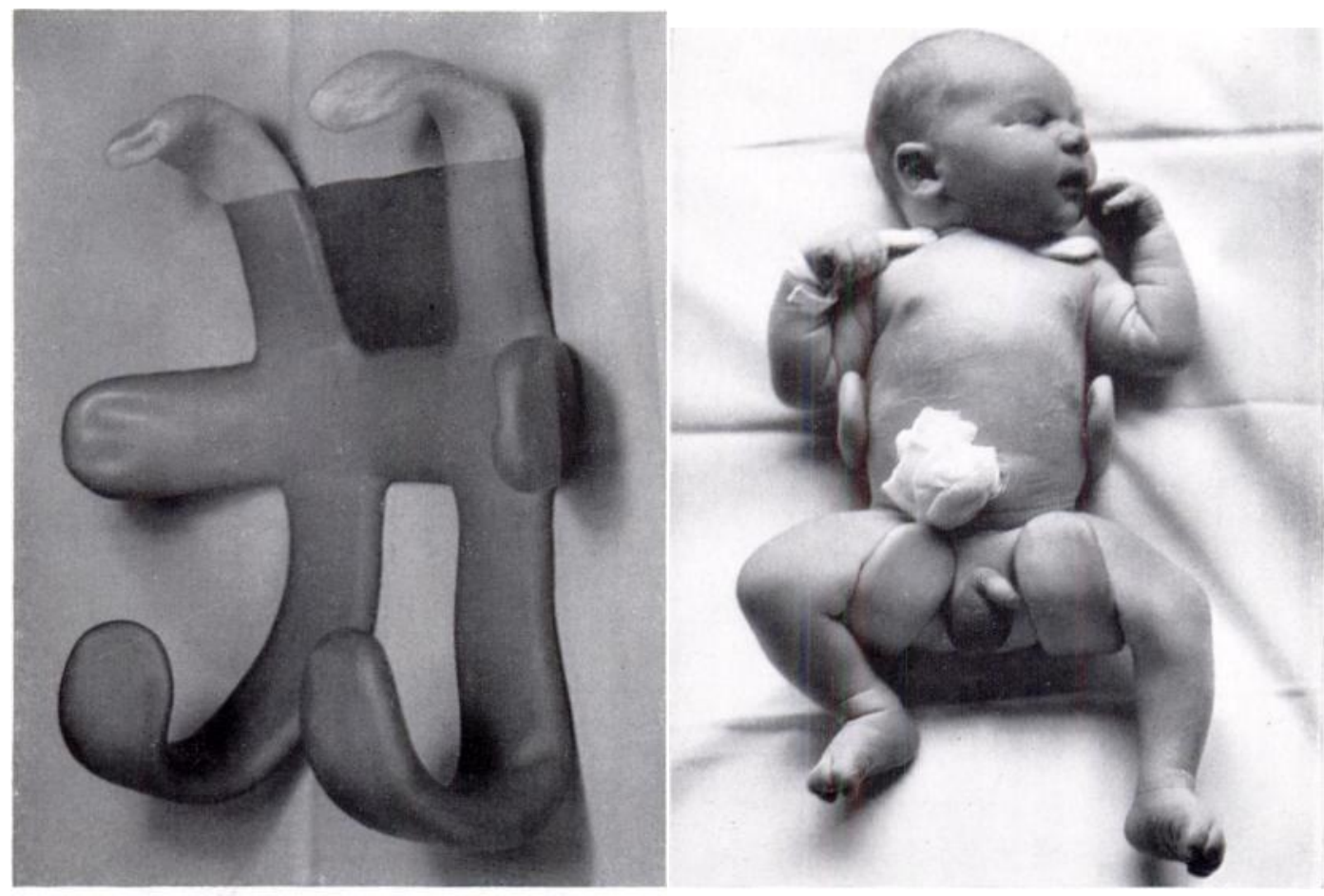

FIG. 3

The light-weight dorsal aluminium splint, used since 1956, keeps the hip joints in the reduced position. It is fitted with a rubber covering. The child can be tended by the mother and washed without being taken out of the splint.

\section{JOINT LAXITY IN CONGENITAL DISLOCATION}

When the dislocated hip joints have been reduced within a few days of birth and held reduced we have repeatedly observed that they become stable after a few weeks. Our favourable results have led us to doubt whether the cause of congenital dislocation of the hip is a dysplasia of the joint.

In September 1959 my radiologist colleague Lars Andrén made an interesting observation in a case of dislocation. He found that there was laxity not only of the hip joints of this child but also of the pelvic joints. In films taken in the Lauenstein position with the legs first drawn apart and then pressed towards the midline, the difference in the distances between the ossified upper ends of the femora was 10 millimetres. The difference in the widths of the symphysis pubis was 3 millimetres, whereas in some seventy control cases the average difference was found to be only 1.5 millimetres (Andrén 1959) (Figs. 11 and 12). Similar observations have been made in five other cases. These observations suggest that the cause of congenital dislocation may be laxity of the joint. One may compare this state with the hormone-induced laxity of the pelvic joints during late pregnancy.

Diczfalusy, Tillinger and Westman (1957) demonstrated that during the first week of life the urine of normal infants contains oestriol in rapidly decreasing amounts: neither oestrone nor oestradiol is found.

On the suspicion that babies with congenital dislocation of the hip might have an abnormal hormone pattern, their urine has been analysed in the hormone laboratory of the Malmö General Hospital. These studies are in a preliminary stage, but so far the cases of dislocation 


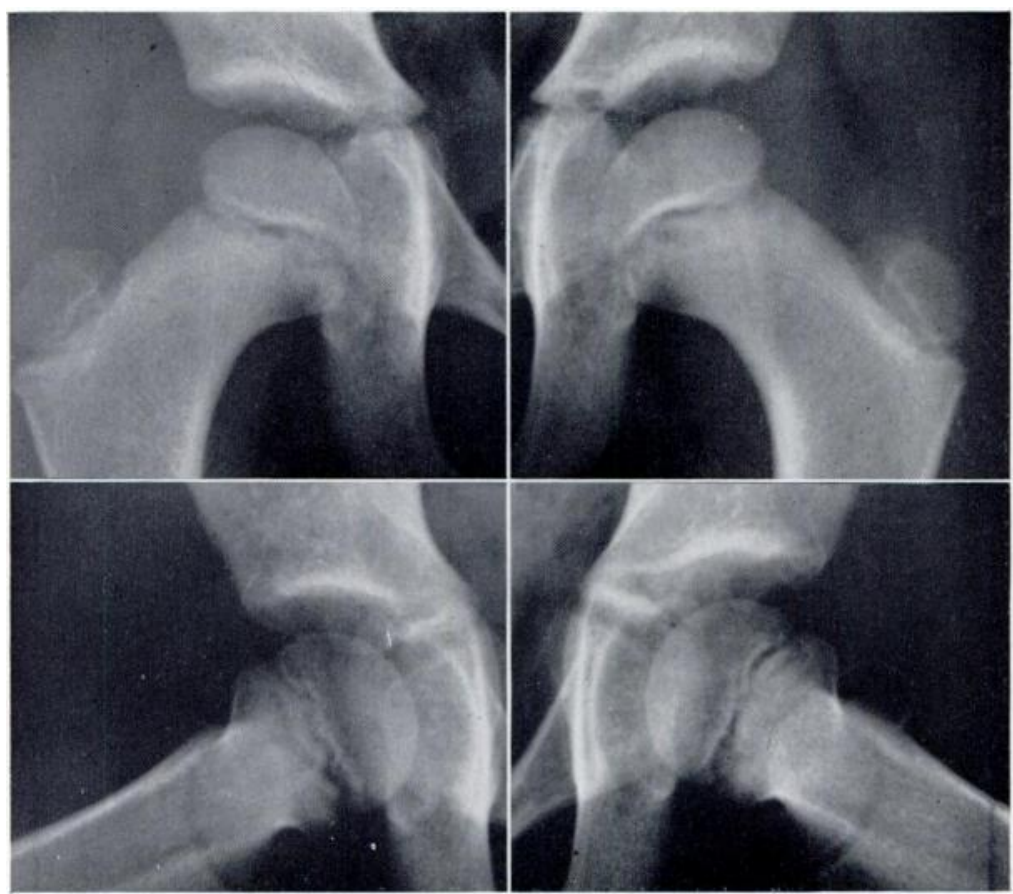

FIG. 4

Radiographs of a girl six years old treated from birth for bilateral dislocation.

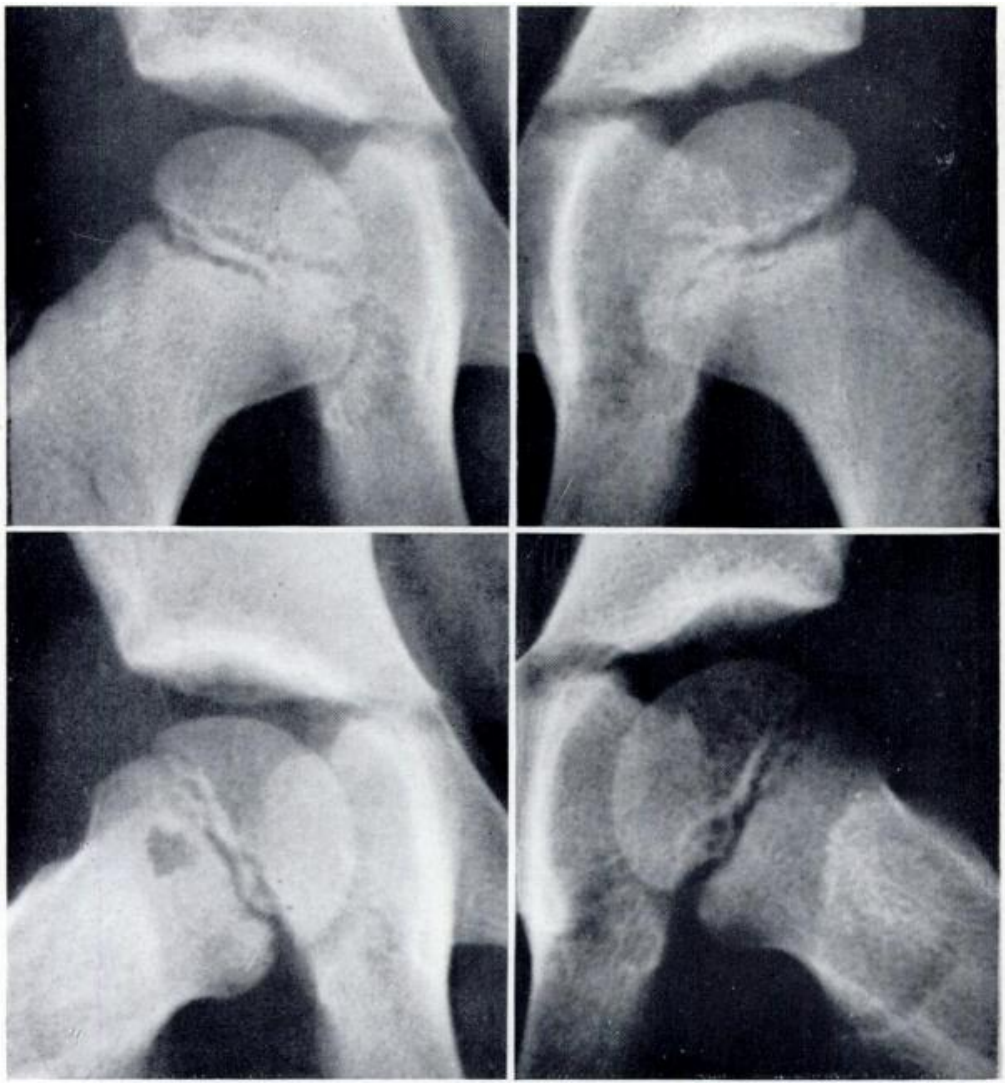

FIG. 5

Radiographs of a girl five and a half years old, treated from birth for bilateral dislocation.

VOL. 44 B, NO. 2, MAY 1962 


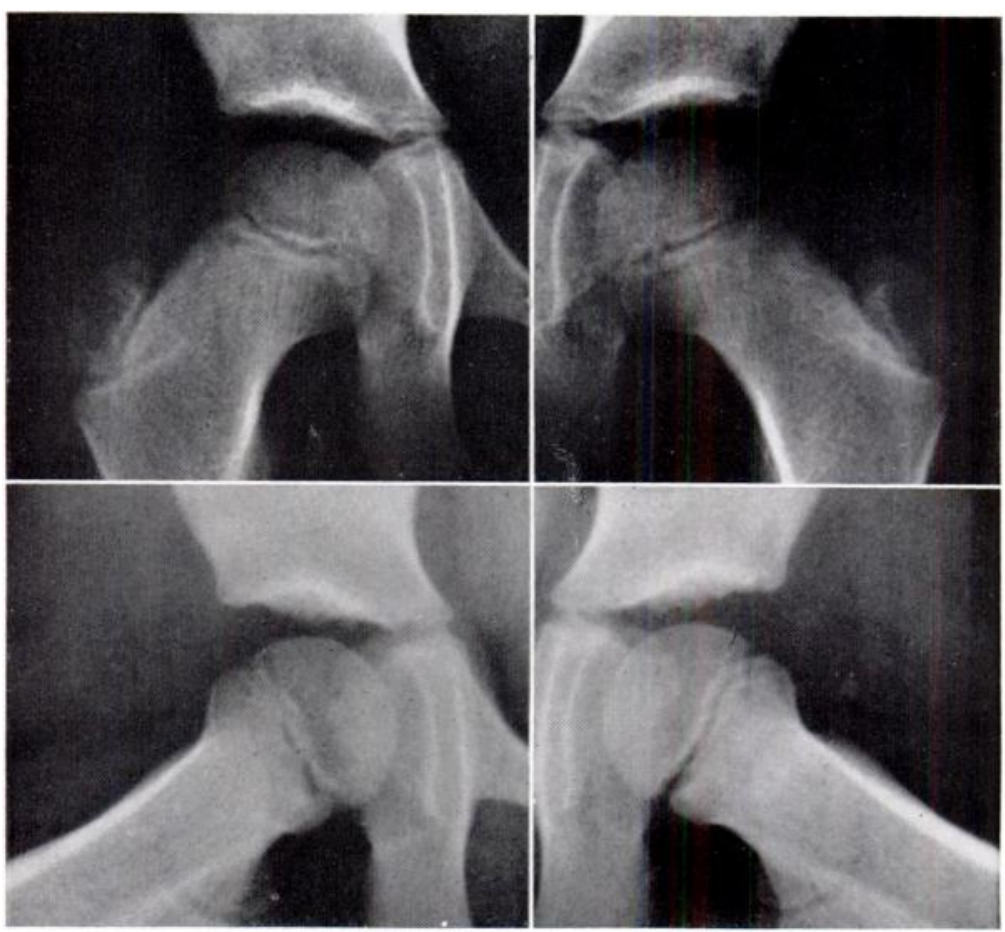

FIG. 6

Radiographs of a girl five and a half years old, treated from birth for bilateral dislocation.

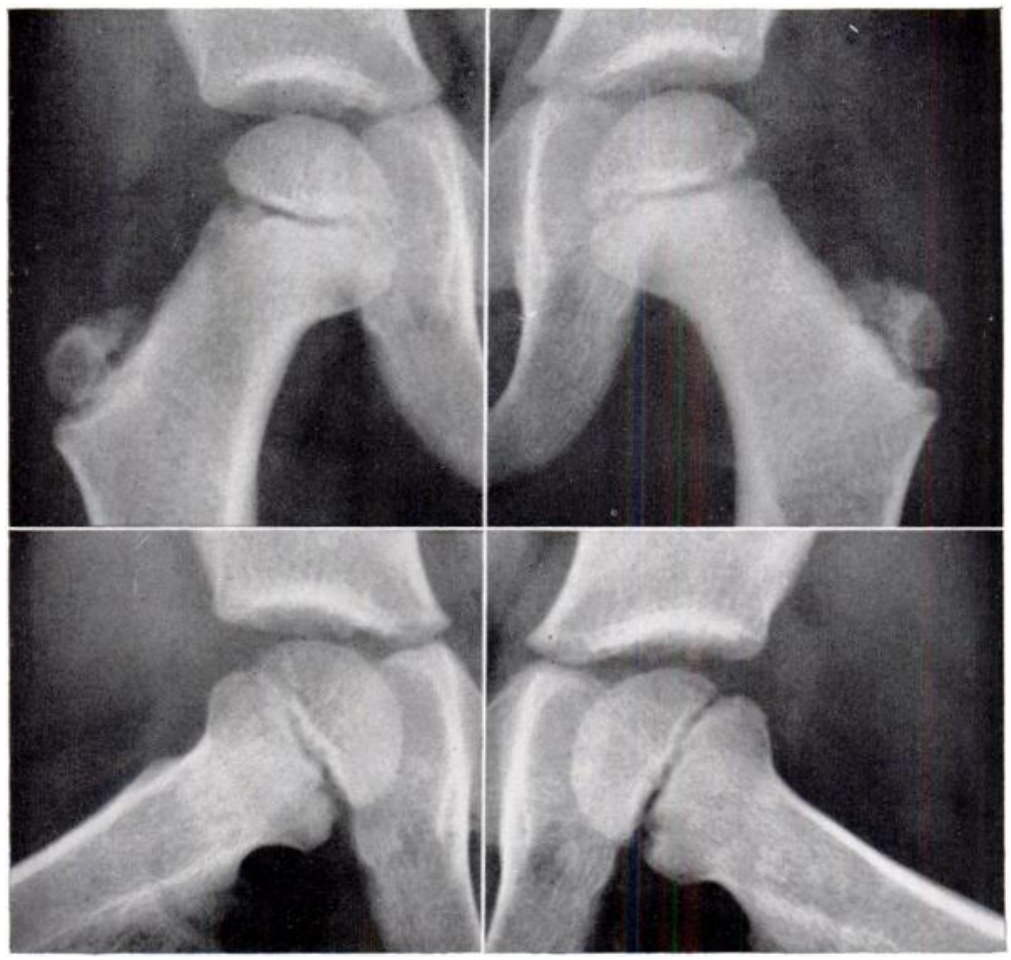

FIG. 7

Radiographs of a girl five and a half years old, treated from birth for dislocation of the left hip. 

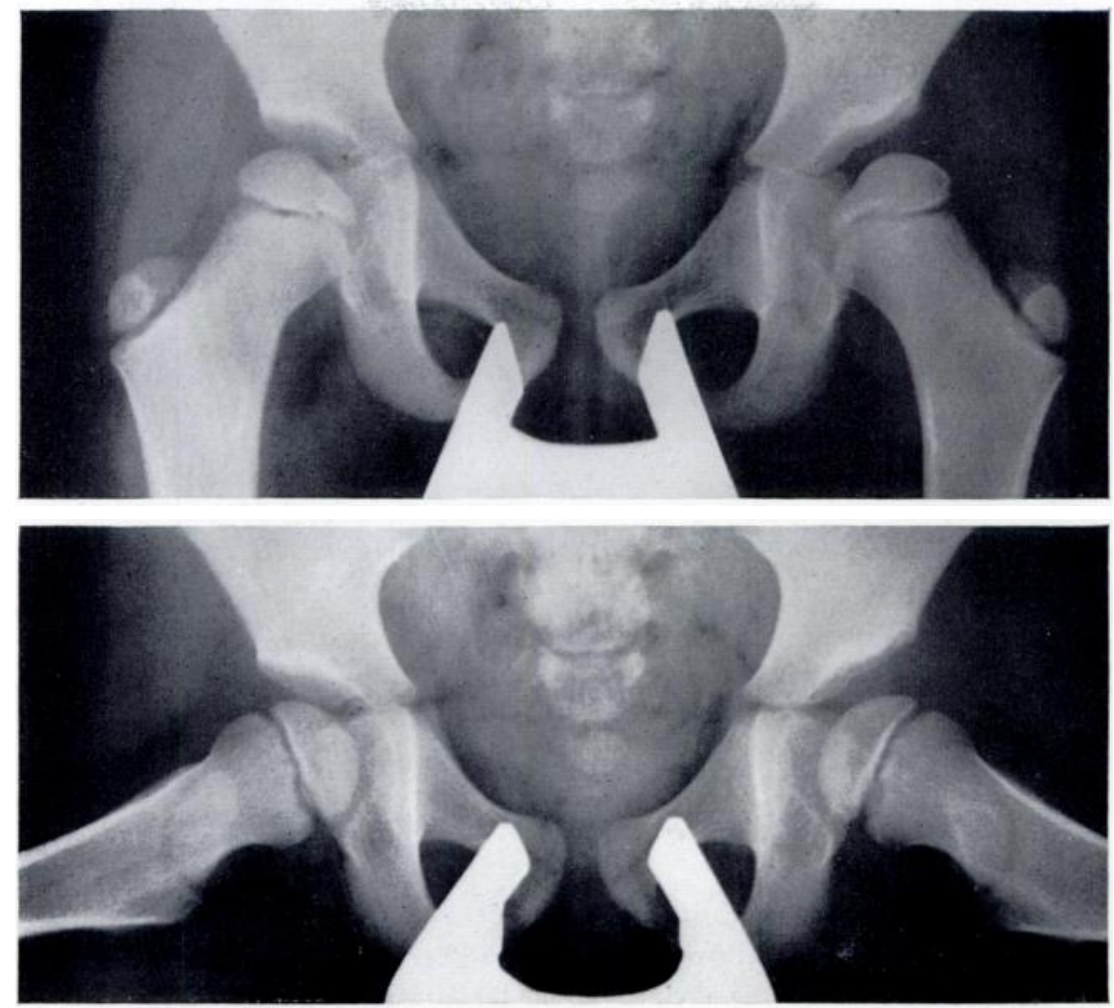

Fig. 8

Radiographs of a girl five years old; bilateral dislocation at birth. The dislocation persisted on the left side, probably because of inadequate treatment during the first five months.

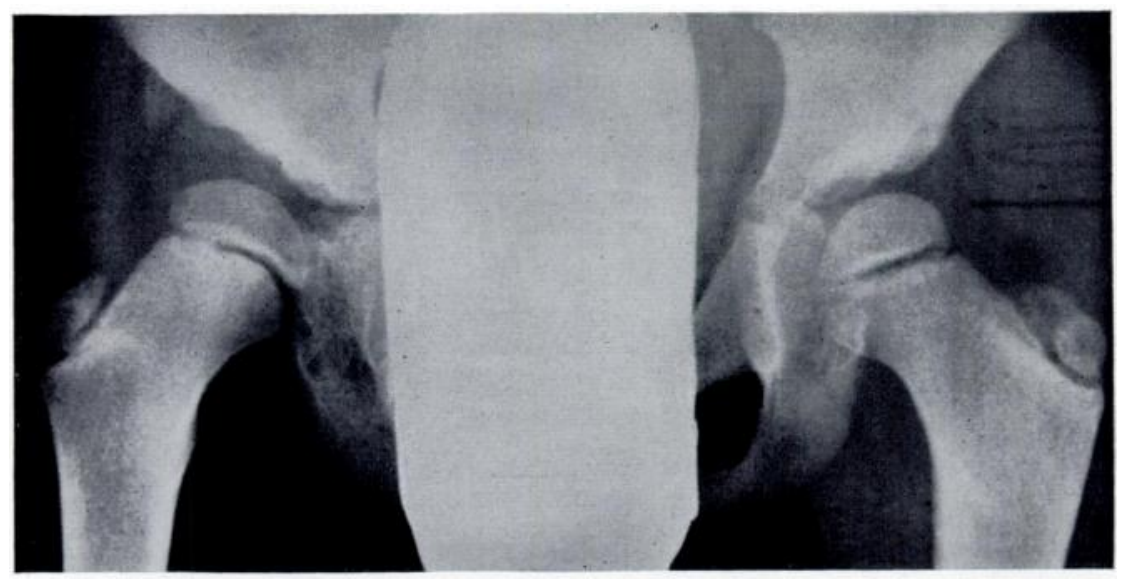

Fig. 9

Radiograph of a girl five years old; bilateral dislocation at birth. The dislocation persisted on the right side, probably because of inadequate treatment during the first eight months. 

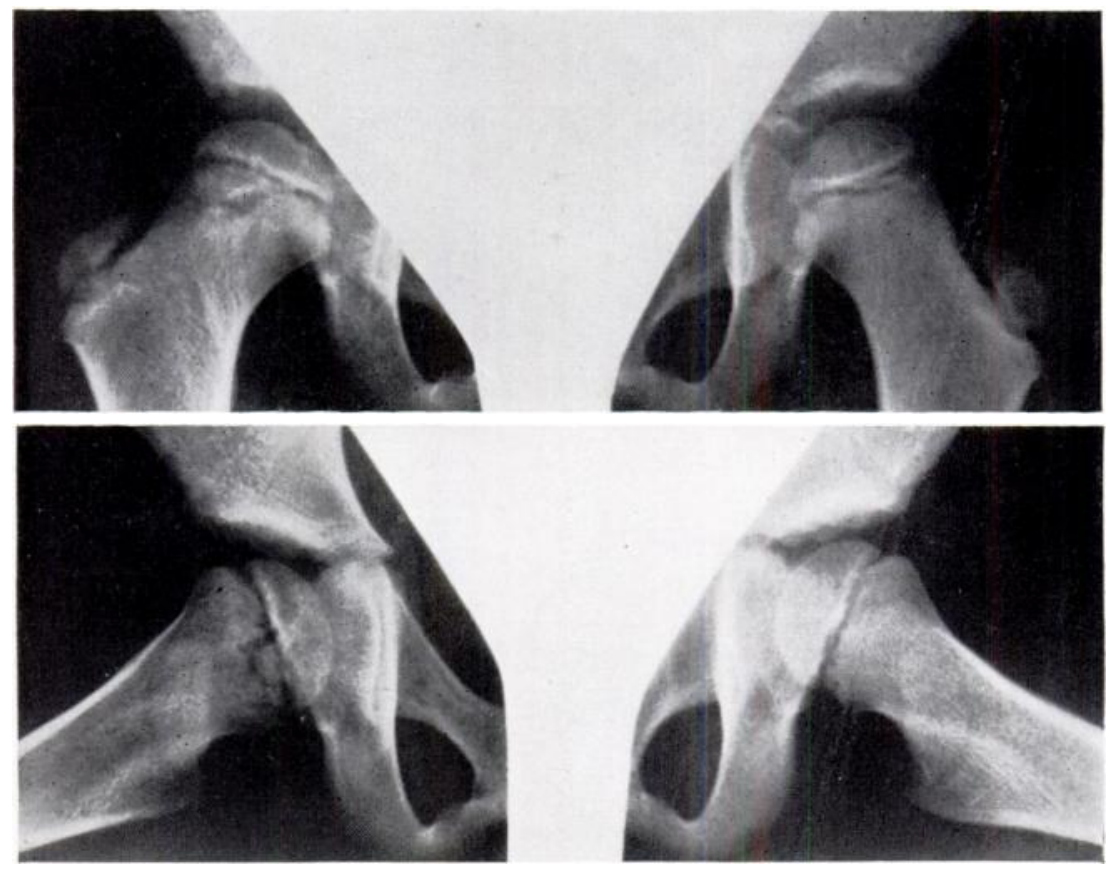

Fig. 10

The radiographs of a girl five years old; bilateral dislocation at birth. Treatment was not started for three weeks and the right hip remained unstable for six months.

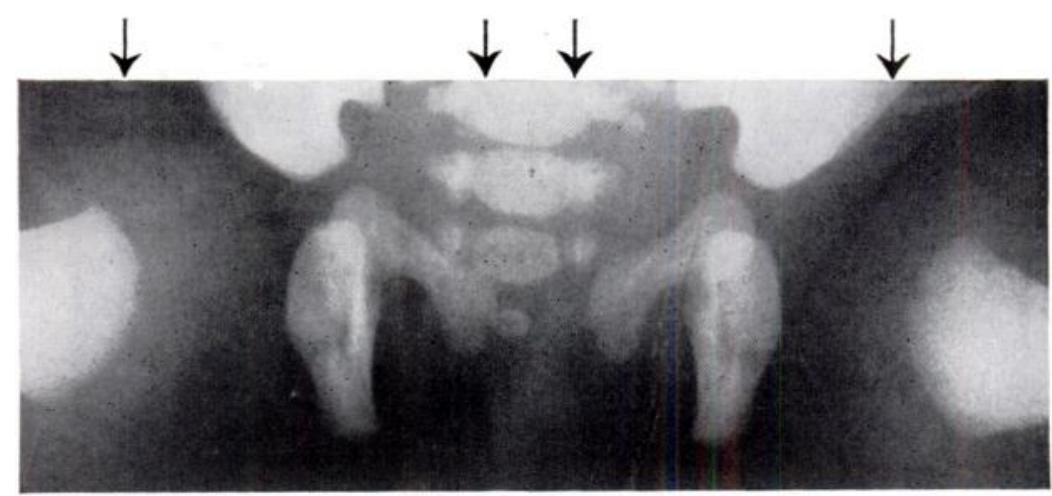

Fig. 11

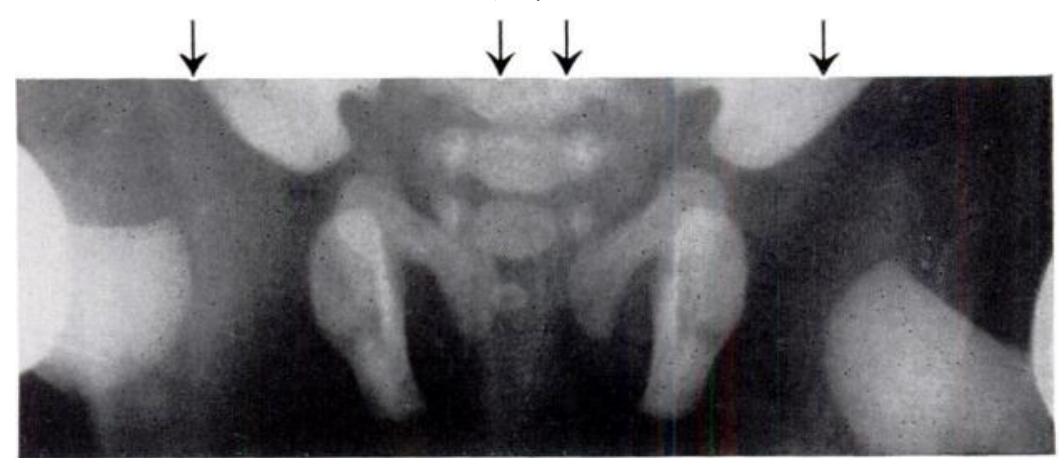

FIG. 12

Radiographs of a new-born girl with bilateral dislocation diagnosed immediately after birth. The radiograph in Figure 11 was made with the legs strongly distracted.

The radiograph in Figure 12 was made with the legs pushed towards the midline. Note the difference in the distances between the proximal ends of the femoral shafts and the difference in widths of the symphysis pubis. 
have shown a hormone pattern differing from that in control cases: the urine has contained not only the normal amount of oestriol, but also a considerable amount of oestrone and oestradiol- $\beta$ (Andrén and Borglin 1961) (Fig. 13). This study is continuing.

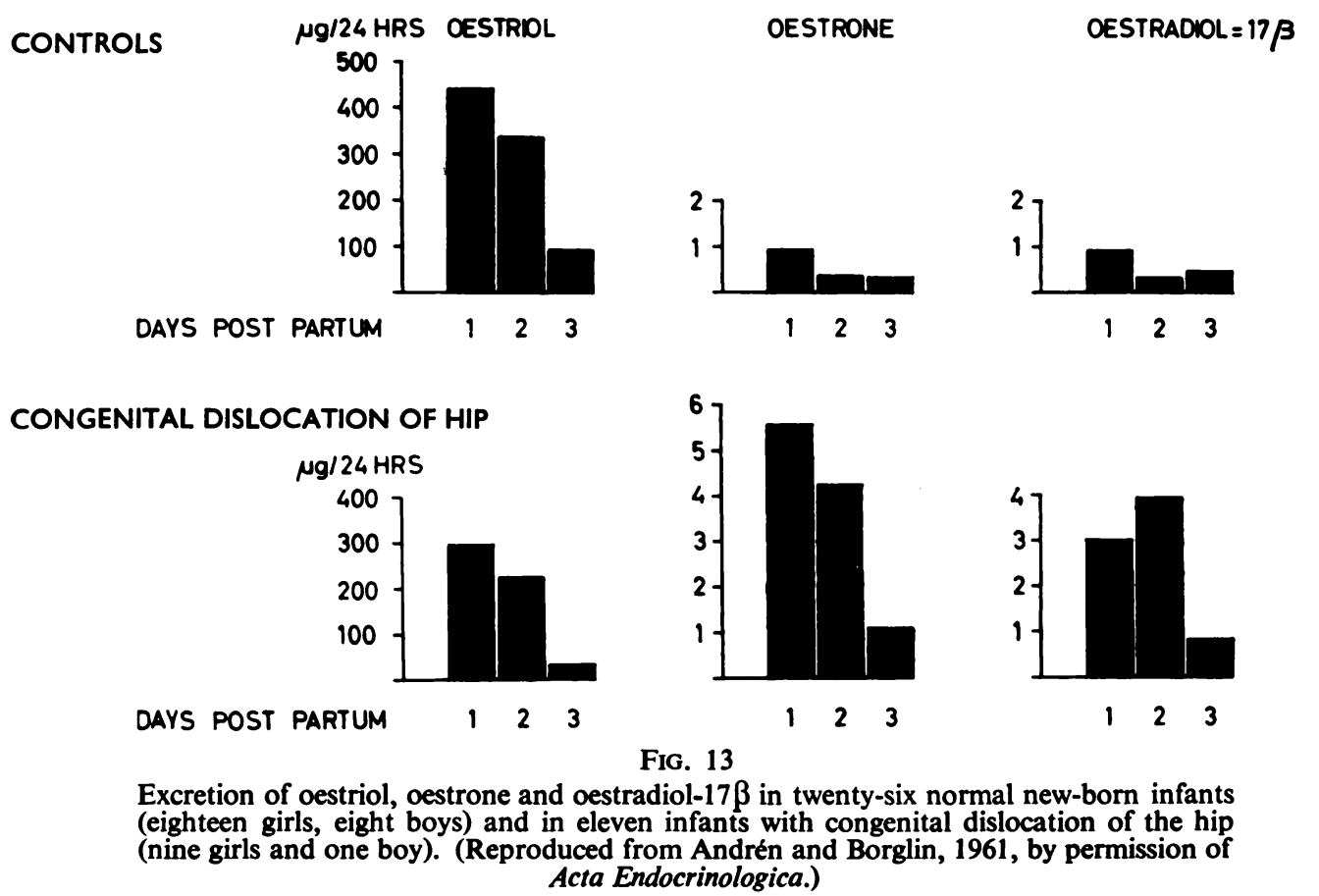

\section{SUMMARY}

1. Congenital dislocation of the hip can be diagnosed clinically immediately after birth and the diagnosis confirmed radiologically by a special technique.

2. If the affected joint is reduced during the first two or three days after birth and held reduced for a period not exceeding three months, the joint will remain stable.

3. This treatment probably leads to normal development of the hip joint.

4. The cause of congenital dislocation of the hip may be laxity of the soft tissues of the joint and not a primary dysplasia of the acetabulum.

\section{REFERENCES}

ANDRÉn, L. (1960): Instability of the Pubic Symphysis and Congenital Dislocation of the Hip in Newborns. Acta Radiologica, 54, 123.

ANDRÉn, L., and BoRglin, N. E. (1961): Disturbed Urinary Excretion Pattern of Oestrogens in Newborns with Congenital Dislocation of the Hip. Acta Endocrinologica, 37, 423.

ANDRÉn, L., and Rosen, S. von (1958): The Diagnosis of Dislocation of the Hip in Newborns and the Primary Results of Immediate Treatment. Acta Radiologica, 49, 89.

Diczfalusy, E., Tillinger, K. G., and Westman, A. (1957): Studies on Oestrogen Metabolism in New-born Boys. 1. Excretion of Oestrone, Oestradiol-17 and Oestriol During the First Days of Life. 2. A Study of the Metabolism of Exogenous Oestradiol-17 $\beta$. Acta Endocrinologica, 26, 303.

Froelich, M. (1911): Traitement de la luxation congénitale de la hanche chez le nourrisson (curatif et préventif). Congrès Français de Chirurgie. Procès-verbaux, 24, $1,048$.

Le Damany, P. (1912): La luxation congénitale de la hanche. Paris: Félix Alcan.

Ortolan, M. (1948): La lussazione congenita dell'anca. Bologna: Cappelli.

Rosen, S. von (1956): Early Diagnosis and Treatment of Congenital Dislocation of the Hip Joint. Acta Orthopaedica Scandinavica, 26, 136.

VOL. 44 B, NO. 2, MAY 1962 